\title{
Acoustic streaming driven enhanced dye-uptake in cells for fluorescence imaging
}

\author{
Melanie E.M. Stamp \\ School of Physics \\ University of Melbourne \\ Melbourne, Australia \\ melanie.stamp@unimelb.edu.au
}

\author{
Christoph Westerhausen \\ Institut für Physik \\ Augsburg University \\ Augsburg, Germany \\ christoph.westerhausen@physik.uni- \\ augsburg.de
}

\author{
Wei Tong \\ School of Physics \\ University of Melbourne \\ Melbourne, Australia \\ wei.tong@unimelb.edu.au \\ Steven Prawer \\ School of Physics \\ University of Melbourne \\ Melbourne, Australia \\ s.prawer@unimelb.edu.au
}

\author{
David J. Garrett \\ School of Physics \\ University of Melbourne \\ Melbourne, Australia \\ dgarrett@unimelb.edu.au \\ Achim Wixforth \\ Institut für Physik \\ Augsburg University \\ Augsburg, Germany \\ achim.wixforth@physik.uni- \\ augsburg.de
}

\begin{abstract}
The major control function in all types of neurons is based on versatile intracellular signals generated by calcium ions. These ions control heart functions as well as the regulation of vital aspects of the entire cell cycle. Calcium imaging is a powerful tool for observing the activity of neurons in neural tissue and for monitoring the effectiveness of electrical stimulation devices. In regard to neuronal prostheses, calcium imagine is used to quantify the electric stimulated interaction between implant and ganglions. Although different techniques exist for staining retinas, most of them lead to cell death or a too low concentration. In this study, we propose to use guided ultrasound to gently sonoporate cells, thus introducing dyes without causing damage. By applying surface acoustic waves we create a microfluidic mixing via acoustically driven chaotic advection and well-defined shear rates with an average of $4000 \mathrm{~s}-1$. We use adherent SaOs-2 cells for a quantitative proof of concept and measured the staining progress in rat retina tissue taken after dye injection into the optical nerve. Our results indicate a $20 \%$ faster dye uptake in cells.
\end{abstract}

Keywords-surface acoustic waves, acoustic streaming, calcium imaging, retina, l cells

\section{INTRODUCTION}

With cumulative knowledge in biomedical science, devices that interact with neural systems such as hearing, vision or movement are in high demand. These devices communicate with the nervous system and have the potential to address some of the most debilitating medical conditions. For example, retinal stimulation devices are of benefit to people with retinal diseases, which results in a loss of photoreceptors within the retina [1]. With these diseases, retinal ganglion cells and other neuronal elements still remain viable and can respond to electrical stimulation. Here, retinal prostheses come to help, were electrode arrays are used to stimulate ganglions inside the retina to restore its function [2-4]. To investigate if cells can be triggered via an electrical signal, calcium imaging techniques are applied [5]. Fluorophores that respond to changes in calcium concentration are introduced into neurons. When neurons fire, the calcium concentration inside the neuron changes dramatically. Thus, neuronal depolarization can be optically detected using these fluorophores. Introducing them into neurons, in particular into whole tissue, is famously difficult as neurons are shocked with large voltage pulses to electroporate the membrane [6,7]. Though effective, this technique results in a high incidence of cell death. A facile method for introducing dye into neuronal tissue without harm is a highly valuable technique in electrophysiology and can have far reaching impact in e.g. targeted drug delivery and uptake in DNA pasmids for viral transfection [8].

A common method in biomedical research and nano-drug delivery is the application of acoustic wave driven micropumping systems for nanoparticle uptake in cells or dissecting tissue $[9,10]$ or ultrasonic, cavitation bubbles to interact and penetrate cell membranes [11-13]. Applying ultrasound to adherent cells, a dynamic pressure is used to open pores in the cell membrane and therefore reach higher loading with desired particles $[8,9]$.

We here present a new approach for neuronal and retinal tissue staining by applying acoustic streaming inside a microfluidic set-up for dynamic a stimulation of cell membranes. Using a modulated and well-defined pressure field with shear rates ranging from $0-4000 \mathrm{~s}^{-1}$, we are able to enhance the fluorescence dye uptake in adherent $\mathrm{SaOs}-2$ cells as a proof of principle. A characterisation of SAW parameters such as heat dissipation has been measured and mechanical 
distortion and electric field, as proven previously [14], are not harming cells or tissue.

\section{Methods And Materials}

\section{A. Cell Culture}

To verify an advance dye uptake in cells, we used the Saos-2 (,sarcoma osteogenic") human osteosarcoma cell line. The cells were cultured in Dulbecco's modified Eagle's medium (DMEM) supplemented with $10 \%$ fetal bovine serum (FPS Superior), $2 \%$ HEPES-buffer $(1 \mathrm{M}), 1 \%$ Lglutamine $(200 \mathrm{mM}), 1 \%$ MEMVitamine with 10mM Na2HPO4 (Biochrom AG, Germany) and $0.2 \%$ Primocin (InvivoGen, France) in NuncTM cell culture flasks (Thermo Scientific,USA) in a saturated atmosphere with $5 \% \mathrm{CO}_{2}$ at $T=37^{\circ} \mathrm{C}$. Cell passaging followed the standard trypsinization procedure using $1 \mathrm{ml}$ Trypsin/EDTA solution and PBS (w/o Ca2+, w/o Mg2+) (Biochrom AG, Germany). The cell density is adjusted to 10.000 cells $/ \mathrm{ml}$.

\section{B. SAW Chip and characterisation}

$\mathrm{LiNbO}_{3} 128^{\circ} \mathrm{Y}^{\prime} \mathrm{Cut}$ was used as piezoelectroc substrate for SAW excitation. An Interdigital Transducer (IDT), being oriented normal to the crystal X-axis is deposited on top of the substrate by standard lithography. It consists of two multifinger electrodes ( $\mathrm{Au}, \mathrm{d}=200 \mathrm{~nm}$ ) with $\mathrm{N}=42$ finger pairs, an aperture of $\mathrm{W}=650 \mu \mathrm{m}$ and electode perioducity of $\mathrm{a}=25 \mu \mathrm{m}$ (wavelength $\left.\lambda_{\mathrm{SAW}}=25 \mu \mathrm{m}\right)$. With the substrate velocity $\mathrm{c}_{\mathrm{LiNbO3} ; 128^{\circ} \text { rot }}$ Ycut $=3980 \mathrm{~m} / \mathrm{s}$, the resonant frequency $\mathrm{f}_{\mathrm{SAW}}=158 \mathrm{MHz}$ is obtained. To ensure bio-compatibility and to protect the metallic structure, the chip is covered with a $200 \mathrm{~nm}$ thick $\mathrm{SiO}_{2}$ layer, deposited via plasma enhanced chemical vapour deposition. The SynthNV RF Signal generator (Windfreak Technologies, LLC) was used to excite the SAW.

To detect possible heat dissipation the temperature rise of the chip under unloaded and water loaded conditions was measured at RT via infrared microscopy. The average shear rate acting on the cells was determined using scanning particle image velocimetry (SPIV), an analysis approach based on the PIVlab toolkit by Thielicke [15-17]. It automatically scans an area larger than a single field of view and combines multiple micro-particle image ( $\mu \mathrm{PIV}$, see [18]). The process is repeated at several heights. This enables the to determinate a three dimensional velocity field. To quantify the shear rates in our set-up, we added latex microbeads $(\mathrm{d}=3 \mu \mathrm{m}$, Polybead, Polysciences
Inc.,Germany) as tracer particles to the cell media solution. A video was recorded using a high-speed camera (FASTCAM 1024PCI, Photron, Germany).

\section{Experimental set-up}

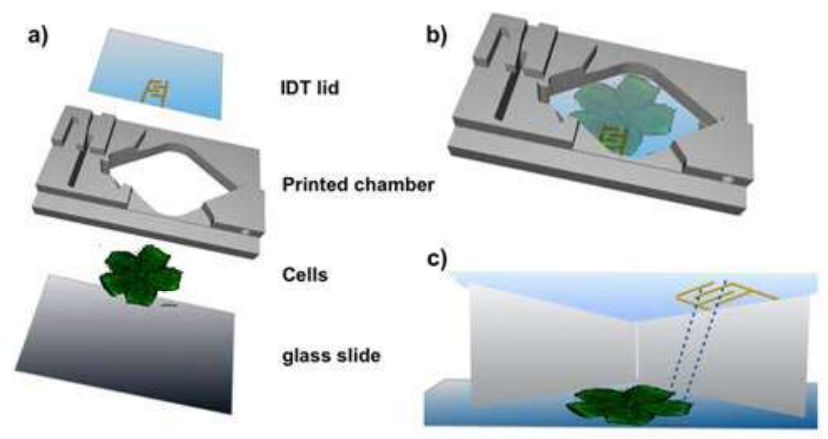

Fig. 1. technical drawing of the experimental set up a) single compartments with cells cultured on a glass slide inside the $3 \mathrm{D}$ printed chamber, the lid is endorsed with the SAW Chip. b) closed chamber and c) inside the chamber showing the acoustic streaming acting on the adherent cell layer.

A fluidic flow system is used as shown in Fig. 1a-c). A 3D printed chamber consiting of polylactic acid is glued to a glass slide housing the cells. The set-up is designed with the IDT Chip to form the lid. We seeded $1 \mathrm{ml}$ of the cellsuspension, filled the whole chamber with nutritive fluid and incubated the cells for $1 \mathrm{~h}$, giving them enough time to adhere to the glass slide. We then added 1 $\mu \mathrm{L} / \mathrm{mL}$ of $1 \mu \mathrm{L}$ calcein green acetoxymethyl fluorescent dye (Invitrogen ${ }^{\mathrm{TM}}$, Thermo Fisher Scientific GmbH, Germany) dissolved in $1 \mu \mathrm{L}$ dimethyl sulfoxide to the cell suspension. In living cells, the acetoxymethyl (AM) esters are removed by intracellular esterase and the dye molecule starts to fluorescence. This way, only living cells become visible during fluorescence microscopy. The device is mounted on a inverted microscope (Axiovert 200M, Zeiss) equipped with a $2.5 \times$ objective and a digital camera (Orca 5G, Hamamatsu Photonics Deutschland $\mathrm{GmbH}$ ). By applying a radio frequency signal to the IDT, SAW were generated, causing an acoustic streaming todwards the cells. An external heating system is applied to monitore a temperature of $\mathrm{T}=$ $37^{\circ} \mathrm{C}$. We performed control experiments under same conditions but without applied RF signal. To monitor the staining process, fluorescence images were taken every $5 \mathrm{~min}$ for $1 \mathrm{~h}$. Images were then analysed using the software ImageJ provided by the NIH, with the cell covered area as a function of time. The time from mounting the sample to switching on the SAW (taking the first image) was taken with $30 \mathrm{~min}$. 


\section{RESUltS AND DisCUSSION}

\section{A. SAW characterisation}

\section{a) Shear rates}

For a detailed determination of local shear rates on the cells and tissue, we applied $\mu \mathrm{PIV}$ at the layer closest to the substrate and at a height of 50 $\mu \mathrm{m}$. In Fig. 2a), we depict the resulting shear map The highest shear rate of $\gamma=4000 \mathrm{~s}^{-1}$ occurs at the position where the stream reaches the substrate and is redirected to the sides and the vertical flow velocity drops to zero. The asymmetric distribution of shear rates is caused by the fact that the jet is tilted relative to the vertical and due to the lateral aperture of the IDT $(\mathrm{W}=650 \mu \mathrm{m})$. The lowest shear rates in the examined area are as low as $\gamma=150 \mathrm{~s}^{-1}$ at the corners of the map.

\section{b) Heat generation}

By generating SAW various losses, such as heat loss appear [19]. To predidct the substrate heating during our experiments, we measured the temperature rise of the used chip under unloaded and water loaded conditions at RT via infrared microscopy. The results are shown in Fig 2b). In the range $P_{I N}=0-100 \mathrm{~mW}$ the temperature increases linearly with increasing power at a rate of $\Delta \mathrm{T} / \Delta \mathrm{P}=37 \mathrm{mK} \mathrm{mW}^{-1}$ for loaded and $\Delta \mathrm{T} / \Delta \mathrm{P}=$ $80 \mathrm{mK} \mathrm{mW}^{-1}$ for unloaded conditions. Applying a power of $\mathrm{P}_{\mathrm{IN}}=15 \mathrm{dBm}$ respectively $32 \mathrm{~mW}$ will lead to a maginal temperature increase of $\mathrm{T}=3 \mathrm{~K}$ during the experiment.
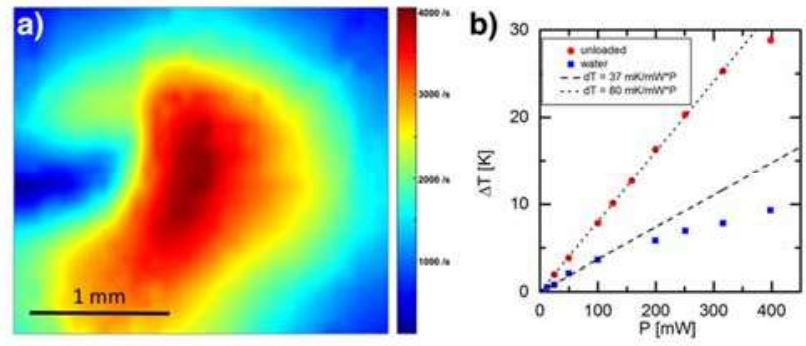

Fig. 3. a) false colour plot of the acoustic straming shear field at the bottom of the chamber. The dark red area marks the indicates the highest shear rates and thus pressure acting on the retina cells. b) temperature increase as function of applied power $P_{I N}$ underunloaded and water loaded conditions.

\section{B. Dye uptake in cells}

Fig. 3. depicts a fluorescence image of the SaOs-2 cells $1 \mathrm{~h}$ after staining with applied SAW (a) and the control sample (b). A visual comparison of the treated and untreated samples clearly indicated an advanced dye uptake due to acoustic streaming.
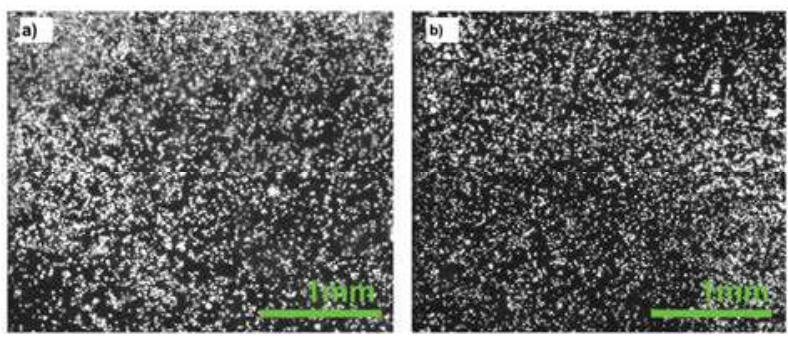

Fig. 2. micrograoh of stained cells after $t=60 \mathrm{~min}$ for a) acoustic streaming treated and b) untreated sample.

To evaluate the scientific content of the images, they were analyzed in terms of the cell area in \% as a function of time T (see Fig. 4). Both graphs show a similar staining at $\mathrm{T}=0 \mathrm{~min}$ with $8.6 \% \pm$ $0.7 \%$ for SAW treated and $8.5 \% \pm 0.8 \%$ for the intreated sample. After $\mathrm{T}=60 \mathrm{~min}$ the stained area

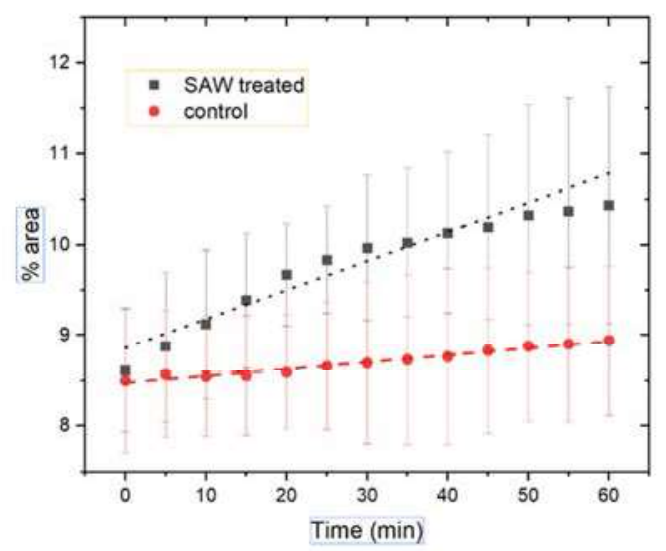

Fig. 4. Plot of staining per \%area as a function of time. With both samples show similar staining at $\mathrm{t}=0 \mathrm{~min}$, the staining rate for SAW treated cells increases with an $16.5 \%$ faster staining rate than the intreated control samnle

with SAW reaches a value of $10.5 \% \pm 1.3 \%$ wereas the staining for the control sample only slitghly increases up to $8.9 \% \pm 0.9 \%$. Our findings show that acoustic streaming has indeed a positive effect on cell staining and leads to a $15.6 \%$ fast dye uptake than by pure diffusion. We yet can't confirm if this effect is driving by the chaotic advection, a targetet pressure field on the cell membrane or a combination of both

\section{CONCLUSION}

In this research, we presented an unconventional method for advance dye uptake. We employed surface acoustic waves and acoustic streaming with well defined shear rates to Saos-2 cells and monitored the staining as a function of time. First studies revealed a successful staining in 
adherent cells exposed to acoustic streaming with a up to $16.5 \%$ higher dye uptake than in control samples.

Our current challenge is to examine the detailed process of acoustic streaming induced dye-uptake using neuronal cells. We here want to investigate if the uptake is driven by the convective streaming itself and therefore a higher dye concentration than through average diffusion or if the shear flow interacts with the cell membrane. Moreover we will continue our studies using retina tissue to find the optimum parameters for fast staining and explore the interaction of direct SAW with tissue.

\section{ACKNOWLEDGMENT}

This work was performed in part at the Melbourne Centre for Nanofabrication (MCN) in the Victorian Node of the Australian National Fabrication Facility (ANFF). This research was supported by the National Health and Medical Research Council (NHMRC) and the Australian Research Council (ARC) through its Special Research Initiative (SRI) in Bionic Vision Science and Technology grant to the Bionic Vision Australia (BVA). Furthermore, the authors thank the "Deutsche Forschungs Gemeinschaft (DFG)" and Nanosystems Initiative Munich (NIM) for the financial support.

\section{REFERENCES}

[1] 1. Hamel, C. Retinitis pigmentosa. Orphanet J. Rare Dis. 2006, 1, 40.

[2] 2. Ganesan, K.; Garrett, D. J.; Ahnood, A.; Shivdasani, M. N.; Tong, W.; Turnley, A. M.; Fox, K.; Meffin, H.; Prawer, S. An all-diamond, hermetic electrical feedthrough array for a retinal prosthesis. Biomaterials 2014, 35, 908-915.

[3] 3. Garrett, D. J.; Tong, W.; Simpson, D. A.; Meffin, H. Diamond for neural interfacing: A review. Carbon N. Y. 2016, 102, 437-454.
[4] 4. Ahnood, A.; Meffin, H.; Garrett, D. J.; Fox, K.; Ganesan, K.; Stacey, A.; Apollo, N. V.; Wong, Y. T.; Lichter, S. G.; Kentler, W.; Kavehei, O.; Greferath, U.; Vessey, K. A.; Ibbotson, M. R.; Fletcher, E. L.; Burkitt, A. N.; Prawer, S. Diamond Devices for High Acuity Prosthetic Vision. Adv. Biosyst. 2017, l, 1600003.

[5] 5. Grienberger, C.; Konnerth, A. Imaging Calcium in Neurons. Neuron 2012, 73, 862-885.

[6] 6. Briggman, K. L.; Euler, T. Bulk electroporation and population calcium imaging in the adult mammalian retina. J. Neurophysiol. 2011, 105, 2601-2609.

[7] 7. Boulling, A.; Escher, P. Coupling ex vivo electroporation of mouse retinas and luciferase reporter assays to assess rod-specific promoter activity. Exp. Eye Res. 2016, 148, 79-82.

[8] 8. Husseini, G. A.; Pitt, W. G. Micelles and nanoparticles for ultrasonic drug and gene delivery. Adv. Drug Deliv. Rev. 2008, 60, 1137-1152

[9] 9. Strobl, F. G.; Breyer, D.; Link, P.; Torrano, A. A.; Bräuchle, C.; Schneider, M. F.; Wixforth, A. A surface acoustic wave-driven micropump for particle uptake investigation under physiological flow conditions in very small volumes. Beilstein J. Nanotechnol. 2015, 6, 414-419.

[10] 10. Nindl I., Toegl A., Sterry W., S. E. High sensitivity and reproducibility of immunohistochemistry with microagitation. Arch Dermatol Res 2004, 296, 278-281

[11] 11. Sajjadi, B.; Raman, A. A. A.; Ibrahim, S. Influence of ultrasound power on acoustic streaming and micro-bubbles formations in a low frequency sono-reactor: Mathematical and 3D computational simulation. Ultrason. Sonochem. 2015

[12] 12. Wu, J.; Nyborg, W. L. Ultrasound, cavitation bubbles and their interaction with cells. Adv. Drug Deliv. Rev. 2008, 60, 1103-1116.

[13] 13. Chen, Y.; Lee, S. Manipulation of biological objects using acoustic bubbles: A review. Integr. Comp. Biol. 2014, 54, 959-968.

[14] 14. Stamp, M. E. M.; Brugger, M. S.; Wixforth, A.; Westerhausen, C. Acoustotaxis - in vitro stimulation in a wound healing assay employing surface acoustic waves. Biomater. Sci. 2016, 4, 10921099.

[15] 15. Thielicke, W.; Stamhuis, E. J. Towards User-friendly, Affordable and Accurate Digital Particle Image Velocimetry inMATLAB. $J$. open Res Softw. 2014, 2

[16] 16. Thielicke, W.; Stamhuis, E. J. Time-Resolved Digital Particle Image Velocimetry Tool forMATLAB (Version: 1.4).

[17] 17. Thielicke, W. The Flapping Flight of Birds-Analysis and Application., Rijksuniversiteit, Groningen, The Netherlands, 2014.

[18] 18. Lindken, R.; Rossi, M.; Große, S.; Westerweel, J. Micro-Particle Image Velocimetry ( $\mu$ PIV): Recent developments, applications, and guidelines. Lab Chip 2009, 9, 2551.

[19] 19. Slobodnik, A. J.; Carr, P. H.; Budreau, A. J. Microwave frequency acoustic surface-wave loss mechanisms on LiNbO 3. J. Appl. Phys. 1970, 41, 4380-4387. 